\title{
Area Under the First Moment Curve Over Dosing Interval
}

National Cancer Institute

\section{Source}

National Cancer Institute. Area Under the First Moment Curve Over Dosing Interval. NCI Thesaurus. Code C85570.

The area under the first moment curve (AUMC) for the defined interval between doses (TAU). 